\title{
DIFFERENCE IN RESPONSE OF SUSCEPTIBLE MOUSE STRAINS TO A SMALL
}

\section{PLAQUE MUTANT (JHM-CC) OF MOUSE CORONAVIRUS}

\author{
Norio Hirano, Tetsuro Tsukamoto*, Satoshi Haga and Yuzo Iwasaki**
}

Department of Veterinary Microbiology, Iwate University, Morioka 020, Japan Department of *Neurology, and $* *$ Neurological Science, Tohoku University School of Medicine, Sendai 980, Japan

\section{INTRODUCTION}

The JHM-CC virus is a small plaque mutant isolated from the DBT cell culture persistently infected with JHM (1), the most neurotropic strain of mouse coronavirus (2). This JHM-CC virus had been shown to induce severe vacuolar degeneration in the brainstem and spinal cord of the infected ICR mice $(1,3)$. Since the strain difference of mice in their susceptibility to various strains of mouse coronaviruses including parental JHM virus has been documented $(4,5)$, we compared the kinetics of virus production and neuropathological changes after intracerebral inoculation of the JHM-CC virus in three strains of mice.

\section{MATERIALS AND METHODS}

Virus. Plaque-purified JHM-CC virus was propagated in DBT cells and infectivity was assayed by the plaque count method as reported previously (2).

Animal inoculation. Four-week-old male Balb/c, C3H and C57BL mice were obtained from a breeder colony, which has been serologically checked for the absence of mouse coronavirus infection. Mice were inoculated intracerebrally (i.c.) with $0.02 \mathrm{ml}$ of virus material.

Infectivity of the brain and spinal cord. Ten percent tissue homogenates (w/v in Eagle's MEM) were prepared from the brain and spinal cord, and assayed for infectivity in DBT cell system as described previously (1).

Immunohistochemical study. Immunohistochemistry was carried out on paraffin sections by the avidin-biotin peroxidase complex (ABC) method using anti-JHM rabbit antibody $(1: 1000)$. For histopathological study, some of serial paraffin sections were stained with hematoxylin and eosin.

\section{RESULTS}

Thirty-one mice each of $\mathrm{Balb} / \mathrm{c}, \mathrm{C} 3 \mathrm{H}$ and $\mathrm{C} 57 \mathrm{BL}$ strains were inoculated i.c. with $1 \mathrm{x}$ $10^{6} \mathrm{PFU}$ of JHM-CC virus, and were observed for the development of clinical signs for 14 days. All the mice became hypersensitive on day 4 to 6 post-inoculation. Among the three strains, $\mathrm{C} 3 \mathrm{H}$ mice were most susceptible to the virus: half of them showed hind leg paresis and began to die on day 8 . The Balb/c mice were most resistant and all survived for 14 days. Some of C57BL mice also showed hind leg paresis and died on day 10 and thereafter. The mortality of mice was about 50\% (15/31) in C3H mice, 20\% (6/31) in C57BL mice and 0\% (0/31) in $\mathrm{Balb} / \mathrm{c}$ mice. At autopsy, marked symmetrical dilatation of the entire ventricular system was the most conspicuous finding in the surviving mice of the $\mathrm{C} 3 \mathrm{H}$ and $\mathrm{C} 57 \mathrm{BL}$ strains. 

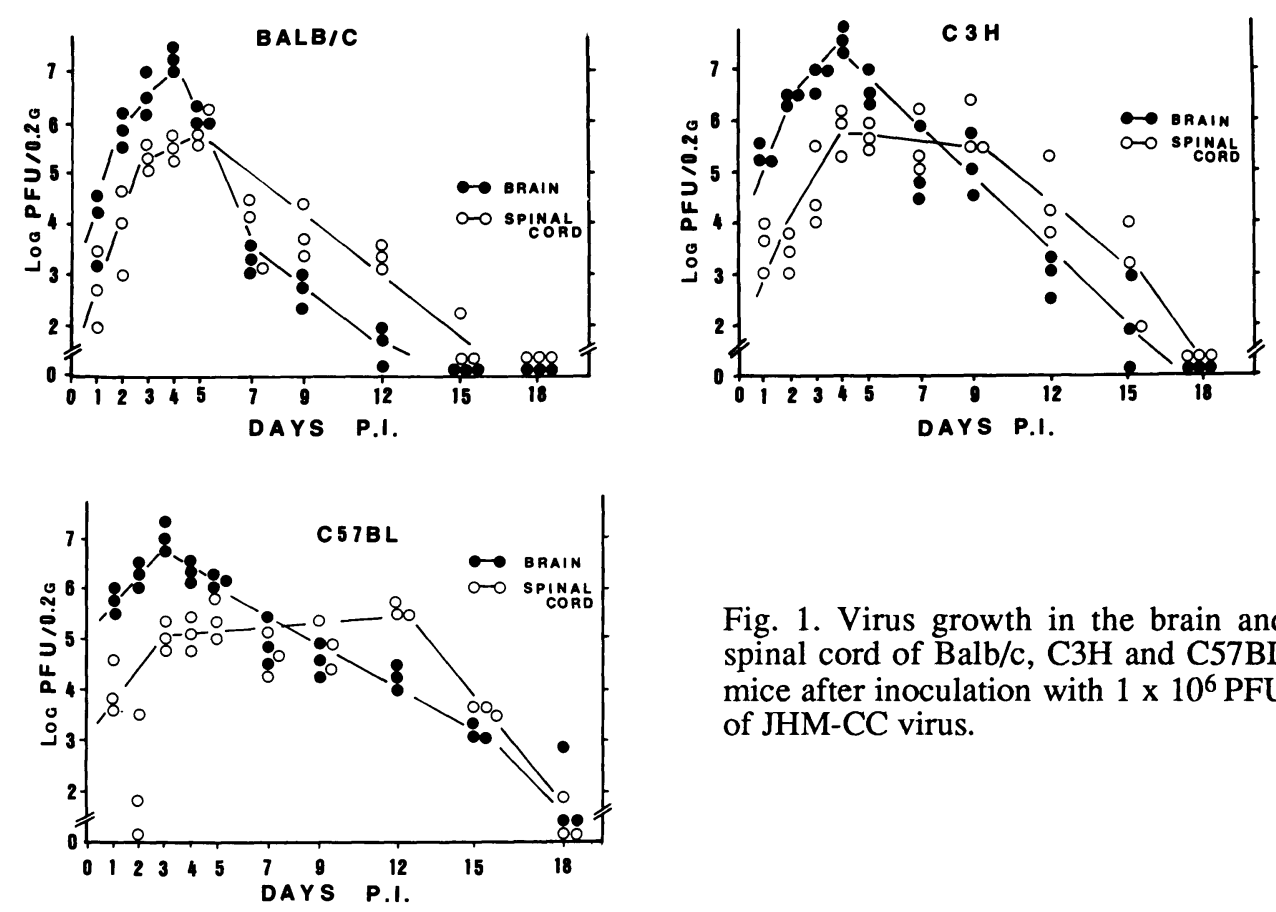

Fig. 1. Virus growth in the brain and spinal cord of $\mathrm{Balb} / \mathrm{c}, \mathrm{C} 3 \mathrm{H}$ and $\mathrm{C} 57 \mathrm{BL}$ mice after inoculation with $1 \times 10^{6} \mathrm{PFU}$ of JHM-CC virus.

To correlate the kinetics of virus titer with histopathological changes in $\mathrm{Balb} / \mathrm{c}, \mathrm{C} 3 \mathrm{H}$ and C57BL mice, 60 mice each of the above strains were inoculated with $1 \times 10^{6} \mathrm{PFU}$ of the virus, and at various intervals, 2 mice each were sacrificed for immunohistochemical study and 3 mice each for virus assay of the brain and spinal cord. As shown in Fig.l, the virus infectivity of the brain reached a peak of $10^{7} \mathrm{PFU} / 0.2 \mathrm{~g}$ on day 3 to 4 . The infectivity gradually declined afterwards and became undetectable on day 18 to 21 . The titer of the spinal cord was 1 to $2 \log$ units lower than that of the brain during first 5 days, and tended to be higher than that of the brain 7 days after inoculation. Among 3 strains, Balb/c mice showed a rapid decline of the infectivity titers, both of the brain and spinal cord, 4 to 5 days after inoculation, and the titers became undetectable by day 15 . On the other hand the infectivity of the brain and spinal cord in C57BL mice was maintained at the detectable level of $10^{3} \mathrm{PFU} / 0.2 \mathrm{~g}$ even 15 days after inoculation. The virus growth in the brain and spinal cord of $\mathrm{C} 3 \mathrm{H}$ mice was almost same to that of C57BL. The virus growth in the brain was parallel with the development of clinical signs as described above.

Using the $\mathrm{ABC}$ method, viral specific antigen was detected on day 3 in the olfactory bulb, cerebrum, brainstem, ependyma, choroid plexus and the aqueduct of Sylvius of the infected mice. Figure 2 shows viral antigens in the ependymal lining cells of the aqueduct of a C57BL mouse sacrificed on day 3. On day 5, virus antigen positive cells were also found in the cerebellum and spinal cord. In C57BL mice, the virus antigen was widely distributed in the CNS, and remained detectable in the cerebral cortex and brainstem even on day 15 when the antigen-positive cells were no longer detectable in the $\mathrm{CNS}$ of $\mathrm{Balb} / \mathrm{c}$ and $\mathrm{C} 3 \mathrm{H}$ mice. On day 3, a stenosis or occlusion of the aqueduct of Sylvius was suspected on the basis of histological examination of C57BL mice. On day 5, enlargement of the lateral ventricle was commonly seen in 3 mouse strains. The enlargement of the ventricles was accompanied by inflammatory cell infiltration and perivascular cuffings of lymphoid cells in the paraventricular tissue. By day 7 , severe vacuolar changes developed in the brainstem of all the infected mice as described previously in ICR mice $(1,3)$. The hydrocephalic change was most remarkable in C57BL mice to compare with that in $\mathrm{C} 3 \mathrm{H}$ and $\mathrm{Balb} / \mathrm{c}$ mice. Figure 1 shows the hydrocephalus in C57BL mouse sacrificed on day 15. 

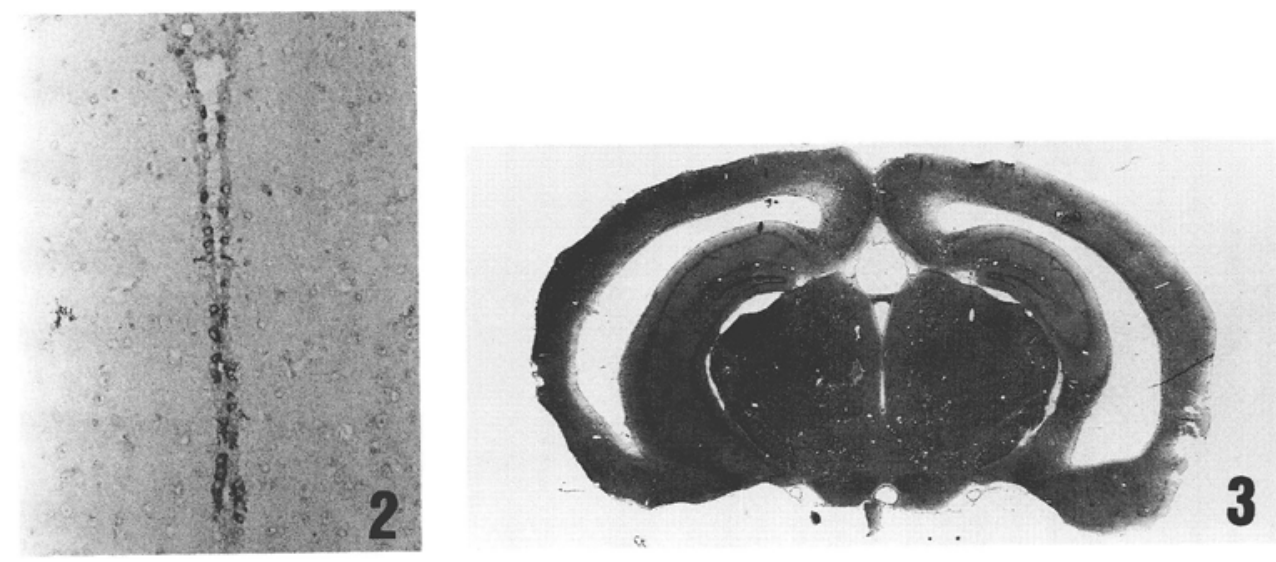

Fig. 2. Viral antigen in the aqueduct of C57BL mouse sacrificed on day 3.

Fig. 3. Hydrocephalus induced in C57BL mouse sacrificed on day 15.

\section{DISCUSSION}

In the present study, $\mathrm{C} 3 \mathrm{H}$ mice were most susceptible to $\mathrm{JHM}-\mathrm{CC}$ virus and $\mathrm{Balb} / \mathrm{c}$ mice were most resistant. Strain difference in susceptibility to the virus was shown by mortality and virus growth in the CNS of each mouse strain. The mortality was considered to be dependent upon the virus growth in the CNS.

As neuropathological changes after virus inoculation, hydrocephalus due to enlargement of lateral ventricle and vacuolar degeneration in the brainstem and spinal cord were found in the infected mice. Viral specific antigen was demonstrated in the ependymal cells, choroid plexus and aqueduct as well as cerebral cortex and brainstem of the infected mice of 3 strains by the $\mathrm{ABC}$ method and a stenosis of the aqueduct was histopathologically suspected at early stage of the infection. Dilatation of the lateral ventricle and aqueduct were found on day 5. On day 7, vacuolar changes were produced in the brainstem and spinal cord and became severe with time, as previously reported on ICR mice $(1,3)$. However, in ICR mice inoculated with the same dose of the virus, no hydrocephalic changes were observed. The highest titer in the brain of ICR mice was $10^{6} \mathrm{PFU} / 0.2 \mathrm{~g}$, and was about $1 \mathrm{log}$ unit lower than that of 3 strains examined in this study. The virus titer and persistence in the brain might play a role in inducing hydrocephalic changes. Hydrocephalus has been induced in experimental animals by a variety of virus infections (6). These experiments show that non-fatal infections with those viruses result in stenosis of the aqueduct leading to hydrocephalus. In the present study, viral growth in the ependyma and choroid plexus was suggested by the $\mathrm{ABC}$ method, and inflammatory cell infiltration was observed in the paraventricular tissue. The JHM-CC virus persisted in the CNS for 2 weeks. These findings suggest that hydrocephalic changes might be produced as a sequence to the stenosis of aqueduct by the virus. Further studies should be made to explain the early pathogenic events in C57BL mice after i.c. inoculation with JHM-CC virus.

\section{REFERENCES}

1. N. Hirano, N. Goto, S. Makino, and K. Fujiwara, Persistent infection with mouse hepatitis virus, JHM strain in DBT cell culture, Adv. Exp. Med. Biol. 142, 301-308 (1981).

2. N. Hirano, T. Murakami, F. Taguchi, K. Fujiwara, and M. Matumoto, Comparison of mouse hepatitis virus strains for pathogenicity in weanling mice infected by various routes, Arch. Virol. 70, 69-73 (1981). 
3. N. Goto, M. Takaoka, N. Hirano, K. Takahashi, and K. Fujiwara, Neuropathogenicity of a mutant of mouse hepatitis virus, JHM strain derived from persistently infecting DBT cells, Jpn. J. Vet. Sci. 46, 755-760 (1984).

4. F. Taguchi, N. Hirano, Y. Kiuchi, and K. Fujiwara, Difference in response to mouse hepatitis virus among susceptible mouse strains. Jpn. J. Microbiol. 20, 293-302 (1979).

5. S. A. Stohlman, and J. A. Flelinger, Resistance to fatal central nevous system disease by mouse hepatitis virus strain JHM. I. Genetic analysis, Immunogenetics 6, 277-281 (1981).

6. R. T. Johnson, Hydrocephalus and viral infections, Dev. Med. Child Neurol. 17, 807-816 (1975). 